\title{
La Revista Mexicana de Ciencias Geológicas a través de los índices de impacto del Institute for Scientific Information
}

\author{
Susana A. Alaniz-Álvarez*, Ángel F. Nieto-Samaniego, \\ Teresa Soledad Medina-Malagón y Ma. Teresa Orozco-Esquivel \\ Centro de Geociencias, Universidad Nacional Autónoma de México, \\ Campus Juriquilla, Querétaro, Qro., 76230. \\ *alaniz@geociencias.unam.mx
}

\section{Resumen}

En este trabajo se analizan los parámetros utilizados para evaluar la calidad de una revista científica y se presenta el caso de la Revista Mexicana de Ciencias Geológicas (RMCG). Se definen y discuten los siguientes índices utilizados por el Institute for Scientific Information: factor de impacto, índice de inmediatez, vida media citada y lista de revistas que la citan. Basados en los valores obtenidos para la RMCG se muestra que su calidad científica es equivalente a revistas de la misma disciplina y categoría que pertenecen al Science Citation Index. Asimismo, se discuten las razones por las que la información descriptiva y la interpretación de la evolución de sistemas geológicos son el objeto tanto de artículos en revistas nacionales como extranjeras, concluyéndose que publicar los datos fundamentales de la Geología mexicana en revistas extranjeras disminuye su impacto en la comunidad nacional, mientras que su impacto a nivel global no es significativo.

\begin{abstract}
In this work we analyze the parameters used by the Institute of Scientific Information in the evaluation of scientific journal quality and present the Revista Mexicana de Ciencias Geológicas (RMCG) as case of study. The impact factor, immediacy index, cited half life and cited journal listing are defined and discussed. Based on the values of these parameters obtained for the RMCG it is shown that the quality of this journal is equivalent to those journals of the same category and discipline. Also it is discussed why the descriptive information and the interpretation of the geological systems are goals of national and international journals of regional Geology. It is concluded that the fundamental data of the Mexican geology published in foreigner journals diminishes their impact in the national community, whereas their worldwide impact is insignificant.
\end{abstract}


Alaniz-Álvarez et al.

\section{Introducción}

En los últimos años, en México se ha insistido a los investigadores que publiquen en revistas incluidas en el Science Citation Index (SCI) elaborado por el Institute for Scientific Information (ISI) (http://www.isinet.com/isi/). Esta institución es un organismo que procura ofrecer una base de datos con las publicaciones más importantes sobre ciencia y con mayor influencia en la investigación actual. El ISI ofrece, a través de suscripción, el acceso a una página web (http://www.isinet.com/isi/products/citation/wos/) donde incluye varios servicios de búsqueda sobre las revistas seleccionadas por sus editores y que aparecen en el SCI. Para determinar la calidad científica de una revista, y así ser candidata a pertenecer al SCI, el ISI considera que la revista debe cumplir, por lo menos, con los estándares de publicación básicos: contar con un comité editorial, que tanto los autores como las referencias incluidas en los artículos sean de diversas nacionalidades, y que los artículos publicados hayan sido revisados y aceptados por especialistas de la misma disciplina.

Para analizar la influencia de las revistas en la investigación, el ISI ha cuantificado por décadas las citas que reciben los artículos de las revistas que pertenecen a su base de datos. Actualmente el SCI expanded (SCIe, en su versión web) contiene más de 5,900 revistas de más de 150 disciplinas científicas. Su base de datos tiene herramientas de búsqueda por autor, por palabras del resumen y título, y por la lista de referencias. Cada año los editores del ISI realizan una revisión de las revistas para ver cuáles de ellas se mantienen y cuáles son eliminadas de su base. Además, analizan la solicitud de cerca de 2,000 nuevos títulos al año, de los cuales aceptan únicamente cerca del 10\%.

En este trabajo se presentan los resultados del análisis de la calidad científica de la Revista Mexicana de Ciencias Geológicas (RMCG) de acuerdo con los criterios que utiliza el ISI. Tanto para la RMCG como para otras revistas de Geología regional incluidas en el SCI, se obtuvo el factor de impacto, el índice de inmediatez, la vida media citada y la lista de revistas que la citan. La búsqueda se hizo durante febrero y marzo del 2003 en la página web del ISI; en esas fechas únicamente estaban disponibles los datos del 2001. Este ejercicio tiene la finalidad de determinar criterios cualitativos y cuantitativos que permitan evaluar la calidad de la RMCG con respecto a publicaciones similares en la comunidad internacional.

\section{Historia de la RMCG}

El Instituto de Geología de la UNAM ha contado con varias publicaciones, entre las que destaca la REVISTA del Instituto de Geología, que ha sido publicada semestralmente desde 1975 y que en 1994 cambió su nombre a Revista Mexicana de Ciencias Geológicas, el cual conserva actualmente. Esta publicación tuvo un cambio muy importante en sus políticas editoriales y en su organización en el año 1998, cuando se convierte en una coedición entre el Instituto de Geología de la UNAM y la Sociedad Geológica Mexicana. La reorganización, tanto en su estructura como en sus políticas editoriales, incluyó la formación de un comité de editores científicos y la publicación de su versión electrónica de acceso libre en http://www.satori.geociencias.unam.mx/RMCG.htm. Actualmente otras sociedades científicas mexicanas se han unido a este esfuerzo editorial, la RMCG es coeditada desde 2002 por tres entidades de la UNAM y tres sociedades geocientíficas, éstas son: Instituto de Geología, UNAM; Centro de Geociencias, UNAM; Facultad de Ingeniería, UNAM; Sociedad Geológica Mexicana A. C.; Instituto Nacional de Geoquímica A. C.; y la Sociedad Mexicana de Paleontología, A. C.

\section{Criterios para evaluar la calidad de una revista científica}

\subsection{Estándares básicos de publicación}

\subsubsection{Puntualidad}

Uno de los aspectos básicos que se considera al evaluar una revista es la fecha puntual de publicación, ya que esto significa que la revista recibe continuamente manuscritos durante el año.

La RMCG tuvo durante gran parte de su existencia un retraso considerable en la fecha de su publicación, sin embargo los últimos ocho números han sido publicados puntualmente (Figura 1). En el último año, el número de artículos sometidos para su publicación se ha incrementado sustancialmente, lo que ha permitido que se cumpla con los tiempos previstos de publicación y que se aumente la periodicidad a tres números por año a partir del 2002.

\subsubsection{Convenciones editoriales internacionales}

De acuerdo con Testa (2003), para que una revista cumpla con las convenciones editoriales internacionales es necesario que:

- el título de la revista sea informativo;

- los títulos de los artículos sean descriptivos;

- cada artículo incluya un resumen;

- la información bibliográfica sea completa en las referencias;

- aparezca la dirección completa de cada autor;

- los títulos de los artículos, las palabras clave y el resumen estén en idioma inglés para facilitar su difusión mundial;

- los artículos publicados hayan sido revisados y aprobado por especialistas de la misma disciplina.

El título Revista Mexicana de Ciencias Geológicas refiere los temas que trata la revista: Paleontología, Geomorfología, Geoquímica, Tectónica, Mineralogía, Vulcanología, Edafología y Geología Regional. 


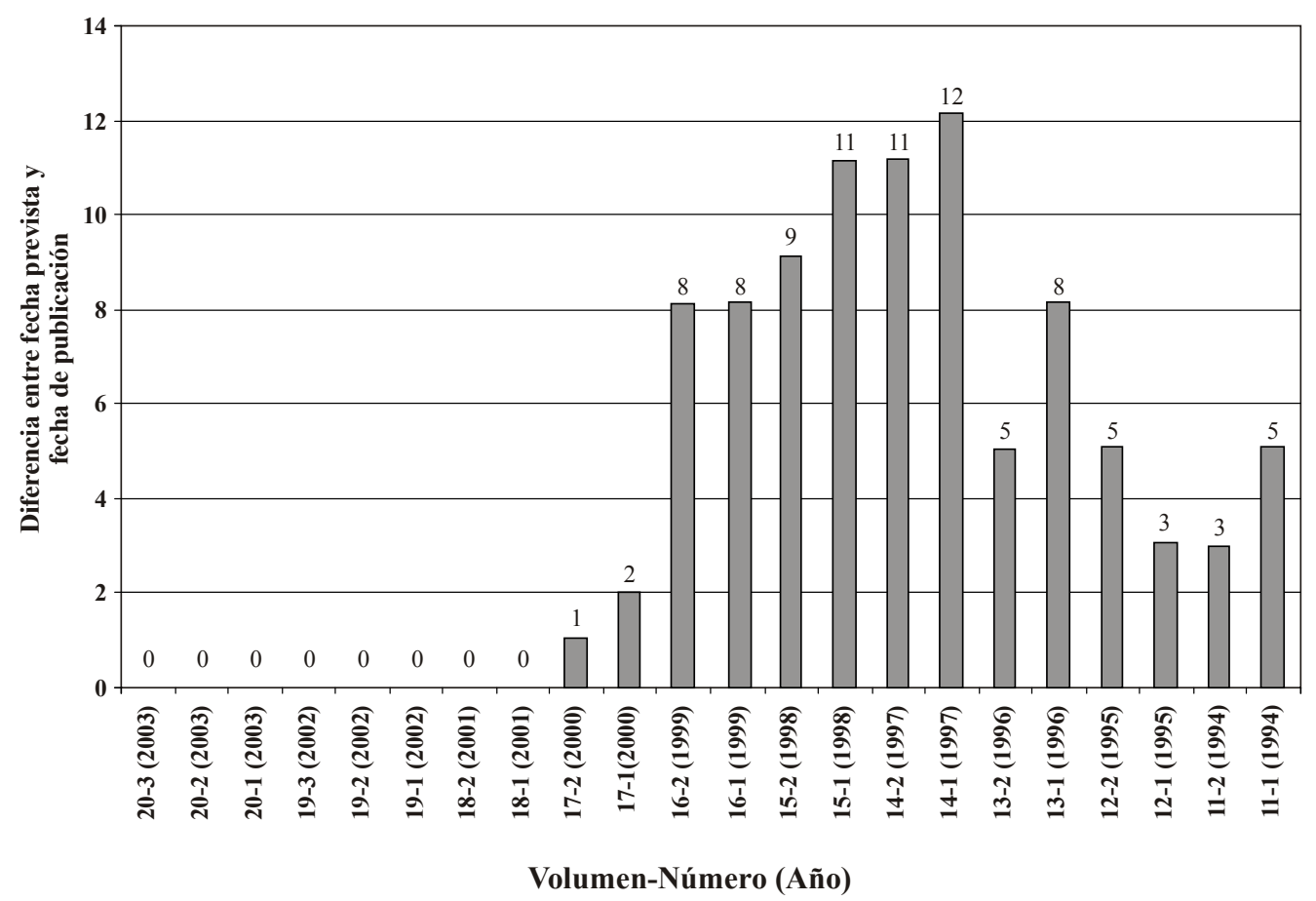

Figura 1. Diferencia entre la fecha prevista y la fecha de publicación de la RMCG, de 1994 al 2003.

Actualmente, esta revista se ha enfocado principalmente a los estudios de la República Mexicana, sin embargo, la RMCG puede ser un medio ideal para publicar el gran cúmulo de información de geología básica sobre Centroamérica y Sudamérica septentrional que no ha sido reportada con los estándares de las revista científicas internacionales. Como en cualquier revista científica, tanto los editores científicos como los encargados de la edición técnica, tienen la encomienda de supervisar que cada uno de los artículos cumpla con los requisitos de claridad descriptiva del título, la inclusión de un resumen, referencias correctamente editadas y las adscripciones de los autores. Estos puntos aparecen en las listas de verificación de cada revista, las cuales se entregan a los revisores de cada artículo y son cotejados por el comité editorial durante el proceso de edición.

En cuanto a la versión en idioma inglés de los títulos de los artículos, los resúmenes y las palabras clave, la RMCG los incluye para todos los artículos; desde 1994 ha publicado el $62 \%$ de los artículos en inglés, el $37 \%$ en español y el $1 \%$ en francés. Se ha mencionado en varios foros que el idioma inglés facilita una mayor audiencia internacional, lo cual es evidente en los tiempos modernos. Sin embargo, si se considera que la inmensa mayoría de la población en la región ocupada por México, el Caribe, Centro y Sudamérica son de habla hispana, la audiencia internacional queda cubierta. El carácter bilingüe de la revistas en Latinoamérica permite una mayor flexibilidad para rescatar el acervo de conocimiento científico que se genera en la zona hispanohablante más grande del planeta.
Que los artículos publicados hayan sido revisados y aprobado por especialistas de la misma disciplina es uno de los requisitos que más atención reciben; todos los artículos de la RMCG han sido evaluados por al menos dos árbitros y únicamente han sido publicados aquellos que tuvieron la aceptación de ambos. Es una idea falsa que las revistas nacionales sólo pueden tener arbitrajes ligeros. Cada cosa que se exponga en los artículos científicos tiene que estar sustentada por datos, por un razonamiento lógico o bien en un trabajo previo publicado en una revista científica. Esto debe aplicarse tanto para revistas regionales o locales, como aquellas de alto impacto y circulación internacional. La diferencia principal entre tipos de revistas radica en el público lector a quién va dirigido el artículo, como se discutirá más adelante.

\subsection{Campo de investigación de la revista}

El ISI resalta que la mayor parte de la literatura científica es cubierta por un número relativamente bajo de revistas, pero en vista de que la investigación avanza rápidamente, nuevos campos de investigación se forman continuamente y por eso es necesaria la adición de nuevas revistas a su base de datos.

Uno de los cuestionamientos que hay que hacer, es si la investigación en geociencias de México ofrece el potencial suficiente para hacer aportaciones novedosas de interés científico regional o global. Nosotros estamos convencidos que sí, la geología mexicana y centroamericana ofrece 
numerosos aspectos que no han sido explicados en la literatura internacional, ya sea por falta de datos (e.g., la ubicación del sur de México en los supercontinentes Rodinia y Pangea, en las reconstrucciones globales; la oblicuidad de un arco volcánico con respecto a la trinchera; la coexistencia de lavas con firmas geoquímicas de distintos ambientes tectónicos; la presencia de una margen continental truncada; el cierre de Centroamérica; la apertura del Golfo de México y su relación con la evolución de Centroamérica y el Caribe), porque los hallazgos recientes pueden aportar elementos críticos a los acervos paleontológicos (e.g., el monstruo de Aramberri; la cantera de Tepexi), o bien, porque ponen en la mesa de discusión teorías científicas, como el gradualismo (e.g., cráter de Chicxulub y extinciones asociadas) o el origen de la Sierra Madre Occidental, que constituye el campo de ignimbritas más grande del mundo.

\subsection{Diversidad internacional}

Al ISI le interesa también que haya diversidad internacional en los editores, autores de los artículos y en las referencias, ya que su interés es la consulta internacional. Se pretende que el SCI contenga en cada categoría un balance global apropiado y, por lo tanto, los editores buscan identificar las mejores revistas regionales que sean de interés de sus suscriptores.

En el SCI, la Geología desarrollada en América del Norte está ampliamente representada, mientras que la de Latinoamérica está subrepresentada. De la primera, en el año 2002 se incluyeron 935 artículos: Canadian Journal of
Earth Sciences (118), Geoscience Canada (18), Geological Society of America Bulletin (116), Geology (312), Bulletin of Canadian Petroleum Geology (32), AAPG Bulletin (137), Canadian Geotechnical Journal (130), Tectonics (72). La parte sur de Norteamérica, Centroamérica y Sudamérica está representada en el SCI por solamente tres revistas, que en el año 2002 incluyeron 142 artículos: Revista Geológica de Chile (14), Journal of South American Earth Sciences (70), y Ameghiniana, revista de la Asociación Paleontológica Argentina (58).

La publicación continua de la RMCG desde 1975 con autores mayoritariamente mexicanos (Figura 2) indica que hay una actividad de investigación geológica en México suficiente para sustentar esta revista. Por otra parte, si se considera que falta mucho para establecer una base sólida de datos geológicos y paleontológicos de México, el Caribe, Centroamérica y Sudamérica, se hace necesario impulsar políticas que promuevan la publicación de esta información.

Los estándares internacionales de edición científica en la RMCG han sido implementados desde hace varias décadas. Sin embargo, existe una falta de reconocimiento de su alto rigor científico por parte de la comunidad científica nacional e internacional.

Uno de los aspectos que más se ha criticado a las revistas regionales o a aquellas que pertenecen a pequeñas sociedades científicas o a universidades, es su distribución local. Es muy difícil para ese tipo de revistas competir con las grandes empresas editoriales, o bien con las sociedades científicas de países desarrollados, las cuales superan por mucho en tamaño y recursos a las sociedades científicas de países como México. Para resolver este problema se decidió que la RMCG fuera auspiciada por sociedades científicas e

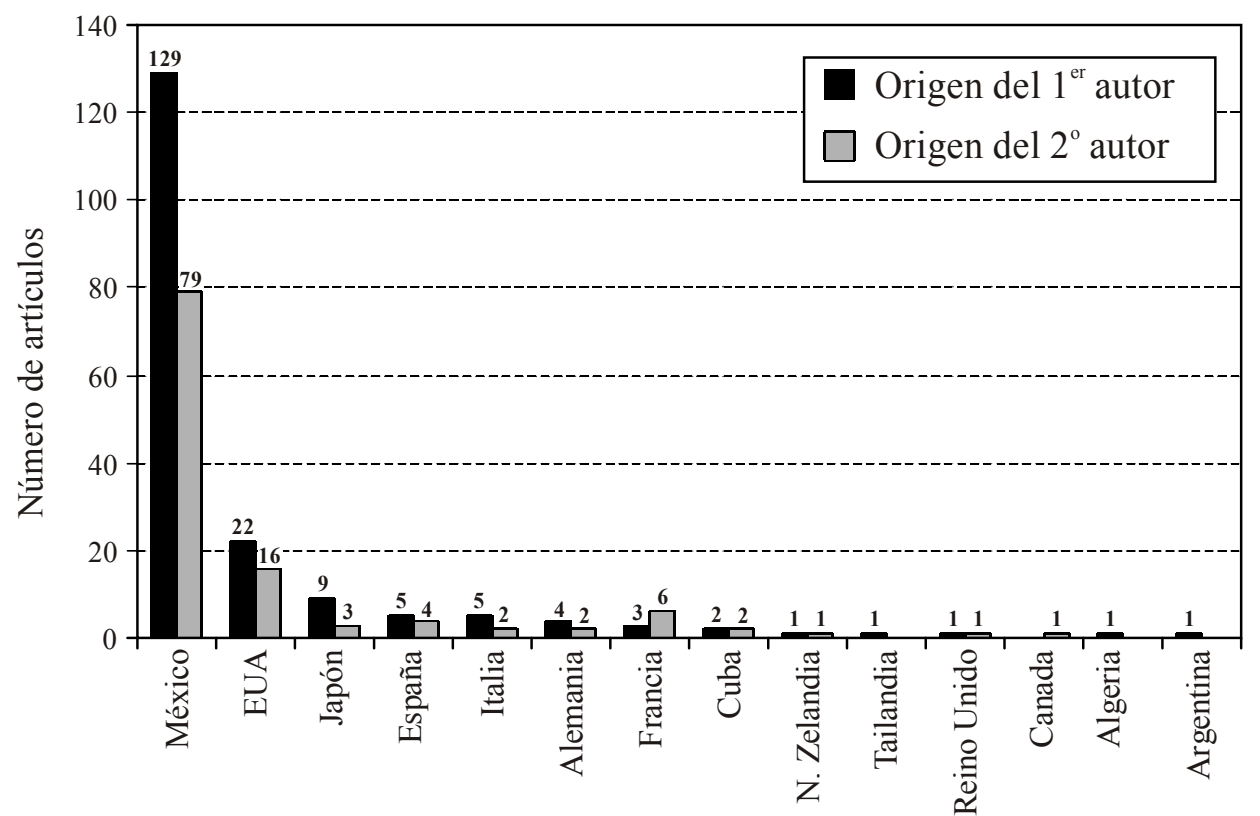

Figura 2. País de origen de los autores. Datos obtenidos de 1994 al 2002. 
instituciones de educación y de esta manera puede ser ofrecida por la Internet con acceso libre; adicionalmente, ha sido incluida en varios índices internacionales como: Actualidades Latinoamericanas, ASFA (Aquatic Sciences and Fisheries Abstracts), Biological Abstracts, Chemical Abstracts, GeoMex, GeoRef, LATINDEX, Periódica (Índice de Revistas Latinoamericanas en Ciencias), Zoological Record. Con lo anterior, el contenido de la RMCG puede llegar gratuita y fácilmente a todo aquel interesado en la Geología de México, en cualquier parte del mundo.

\subsection{Análisis de citas}

Las citas representan el reconocimiento formal que hace un autor a la investigación publicada anteriormente; ésta queda registrada públicamente en la lista de referencias. El análisis de las citas determina, de alguna manera, cuáles son las publicaciones que más han influido en la investigación.

Se llevó a cabo la búsqueda de citas de la RMCG en el SCI y por otro lado se le pidió a gran parte de los autores que nos enviaran las citas a sus trabajos no incluidas en el SCI. El análisis que aquí presentamos abarca desde 1994 hasta el 2002. De todas las citas capturadas, el $70 \%$ proviene de revistas que pertenecen al SCI y el $30 \%$ restante a revistas no incluidas ahí. Este último valor debe de considerarse como un mínimo ya que únicamente se le solicitó la relación de sus citas al personal académico de la UNAM que ha publicado en la RMCG.

Desde 1994 se han publicado 184 artículos en nueve volúmenes. La RMCG ha sido citada 221 veces (SCI + noSCI), esto quiere decir que el promedio de citas que ha recibido cada artículo de la RMCG es de 1.2. De los cinco volúmenes más citados (Figura 3), incluyendo las citas del SCI más las no incluidas en el SCI, tres corresponden a volúmenes especiales: el 12-2, editado por Gloria Alencáster y Blanca Estela Buitrón-Sánchez (Conferencia Internacional sobre Rudistas, con 57 citas), el 14-2 editado por George D. Stanley Jr. y Carlos M. González-León (International Workshop on the Geology of Northwestern Sonora, Mexico, con 29 citas) y el 15-2 editado por Gerardo de Jesús Aguirre Díaz (Tectonic and magmatic evolution of Mexico during the Cenozoic, con 20 citas). El volumen regular que más citas por artículo ha recibido es el 13-2 (Figura 4).

Cabe destacar que el trabajo más citado corresponde a una contribución de la paleontología del Caribe "Stratigraphy, composition and age of Cuban rudistbearing deposits" por Reynaldo Rojas (RMCG, 1995, v. 12-2, p. 272). El segundo trabajo más citado es "Volcanic stratigraphy of the Amealco caldera and vicinity, central Mexican Volcanic Belt" por Aguirre-Diaz, G. J. (RMCG, 1996, v. 13-1, p. 10) y el tercero "Estructura interna de la falla de Oaxaca (México) e influencia de las anisotropías litológicas durante su actividad cenozoica" por NietoSamaniego et al. (RMCG, 1995, v. 12-1, p. 1). De los trabajos más citados se observa que existe diversidad en los temas tratados, el tipo de volumen (especial o regular) y la nacionalidad de los autores; esto sugiere diversidad en el público lector de la RMCG.

El número de citas depende principalmente de dos factores: del número de investigadores que hagan trabajo en el mismo campo (e.g., Sylvan-Katz, 1999) y de la distribución de la revista. Si la RMCG incorpora en su campo de estudio a Centro y Sudamérica, el primer factor se incrementará sustancialmente. Desde principios del 2002, la revista en papel se distribuye entre los miembros de las sociedades geocientíficas involucradas en su edición, y la tabla de contenido y el texto completo se encuentra en línea

\section{Citas por Volumen}

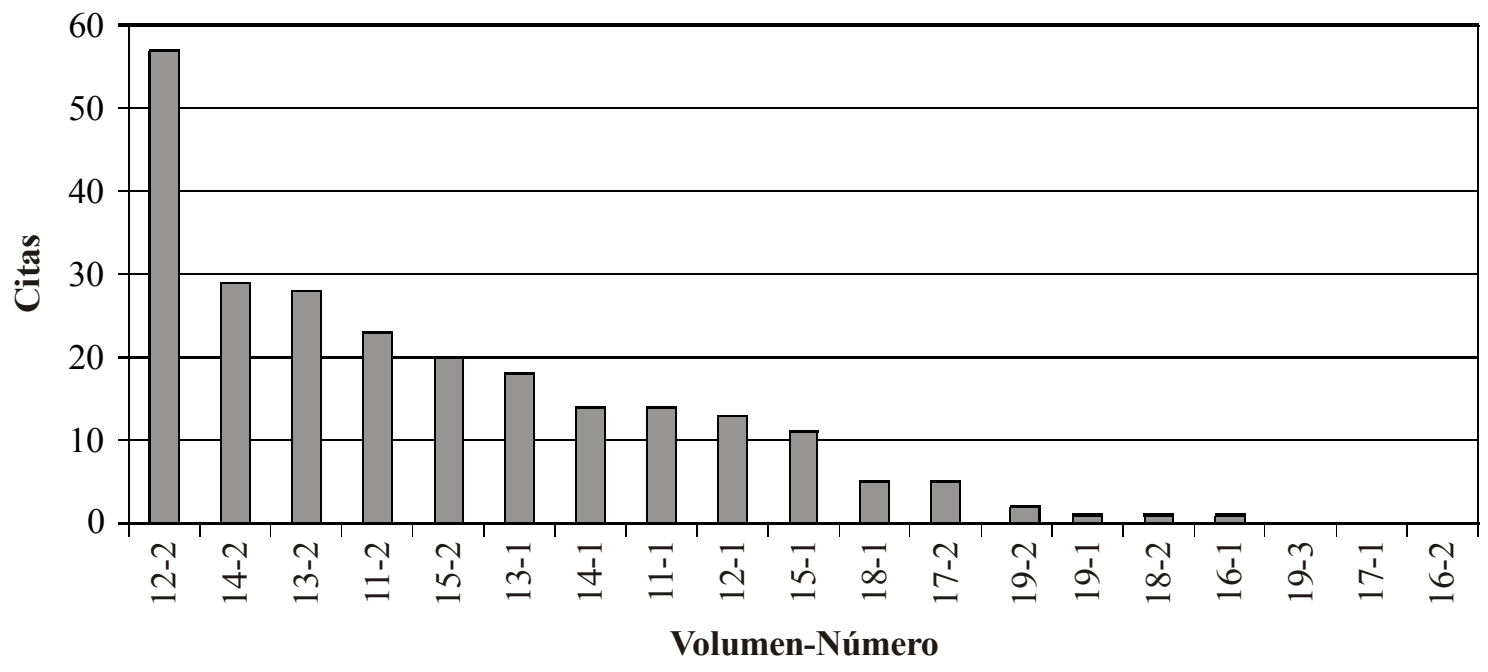

Figura 3. Volúmenes más citados de la RMCG de 1994 al 2002. Nótese que no están en orden cronológico. 


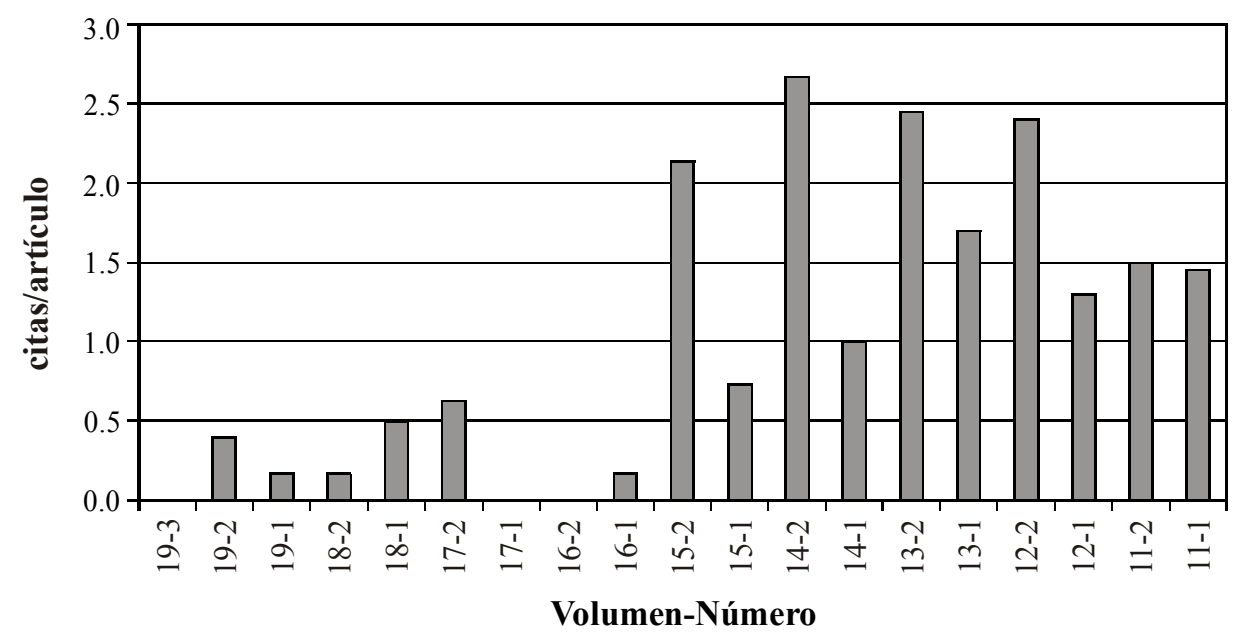

Figura 4. Número de citas normalizadas por artículo de la Revista Mexicana de Ciencias Geológicas de 1994 al 2002.

en la dirección web: http://www.satori.geociencias.unam.mx /RMCG.htm. Estas dos acciones pretenden aumentar el número de lectores y por lo tanto el impacto de la revista a nivel internacional.

Varios índices se han establecido para evaluar el alcance de las revistas. Para cada revista incluida en su base de datos, el ISI captura el número total de citas recibidas en cada año, la vida media citada, la lista de revistas que la citan, vida media de la citación y la lista de las revistas que más son citadas por dicha revista. En este trabajo no fueron obtenidos los últimos dos parámetros ya que no contamos con los datos necesarios.

\subsubsection{Factor de impacto}

El factor de impacto (FI) es una medida de la frecuencia con la cual el artículo promedio de una revista ha sido citado en un año en particular. El factor de impacto se calcula dividiendo el número de citas a los artículos publicados en los dos años previos, que aparecen durante el año a evaluar, entre el total de artículos publicados en los dos años previos.

Se calculó el factor de impacto de la RMCG para el 2002 de la siguiente manera:

Citas en 2002 a artículos publicados en

$2001=2$

$2000=4$

$\mathrm{A}=$ Total de citas en 2001 y $2002=6$

$$
\text { Factor de impacto }=\mathrm{A} / \mathrm{B}=0.23
$$

Este factor ayuda a evaluar la importancia relativa de una revista, especialmente cuando se compara con otras de la misma disciplina y de la misma categoría. La Tabla 1 y la Figura 5 comparan el factor de impacto 2002 de la RMCG con el de otras revistas de Geociencias pertenecientes al SCIe para 2001, pero que no fueron publicadas por empresas editoriales. La razón de comparar con el FI para 2001 es que a la fecha de elaboración de este trabajo, aún no estaban accesibles los FI 2002 de las revistas SCIe referidas.

La RMCG tuvo un FI para el 2000 y el 2002 casi del doble de lo obtenido en el segundo lustro de los años 90. Aunque aparentemente el FI es bajo, es semejante al que tienen otras revistas de la misma categoría (Figura 5).

\subsection{2. Índice de inmediatez (Inmediacy Index)}

El índice de inmediatez es una medida de qué tan rápido el artículo promedio en una revista es citado. Este índice mostrará con que frecuencia los artículos de una revista son citados en el mismo año de publicación. Este índice se calcula dividiendo el número de citas a artículos publicados en cierto año, entre el número de artículos publicado en ese año. Por ejemplo, para la Revista Mexicana de Ciencias Geológicas:

Número de citas recibidas en 2002 a

artículos publicados en 2002: 2

Número de artículos publicados en 2002: 25

Índice de inmediatez $2002=0.08$

Número de citas recibidas en 2001 a

artículos publicados en 2001: 2

Número de artículos publicados en 2001: 12

Índice de inmediatez $2001=0.16$

Las revistas con más números por año tienen ventaja para obtener mayor índice de inmediatez, porque un artículo publicado en los primeros meses del año tiene mayor oportunidad de ser citado que los que se publican al final. Para comparar revistas especializadas en investigación de frontera, el índice de inmediatez puede proveer una perspectiva útil de las revistas con más números por año. Esto es evidente cuando se compara el índice de inmediatez de las revistas Geology y Earth and Planetary Science 
La Revista Mexicana de Ciencias Geológicas a través de los índices del ISI

Tabla 1. Comparación del factor de impacto y otros indicadores de Revistas de Geociencias incluidas en el SClexpanded pero publicadas por instituciones no privadas.

\begin{tabular}{|c|c|c|c|c|c|c|c|c|}
\hline Título de la Revista & ISSN & $\begin{array}{c}\text { Total de } \\
\text { citas } \\
(1 \text { año })\end{array}$ & $\begin{array}{c}\text { Factor } \\
\text { de } \\
\text { impacto }\end{array}$ & $\begin{array}{c}\begin{array}{c}\text { Índice } \\
\text { de }\end{array} \\
\text { inmediatez }\end{array}$ & $\begin{array}{c}\text { Artículos } \\
\text { (1 año) }\end{array}$ & $\begin{array}{c}\text { Vida } \\
\text { media } \\
\text { citada }\end{array}$ & Editorial & País \\
\hline $\begin{array}{l}\text { Acta Geologica Sinica- } \\
\text { English Edition }\end{array}$ & $1000-9515$ & 234 & 0.271 & 0.148 & 54 & 7.1 & $\begin{array}{c}\text { Geological } \\
\text { Society of China }\end{array}$ & China \\
\hline Atmósfera & $0187-6236$ & 53 & 0.324 & 0.000 & 17 & & UNAM & México \\
\hline $\begin{array}{l}\text { Ingeniería Hidráulica en } \\
\text { México }\end{array}$ & $0186-4076$ & 34 & 0.093 & 0.078 & 51 & & $\begin{array}{c}\text { Instituto } \\
\text { Mexicano de } \\
\text { Tecnología del } \\
\text { Agua }\end{array}$ & México \\
\hline Resource Geology & $1344-1698$ & 103 & 0.357 & 0.208 & 24 & 3.7 & $\begin{array}{l}\text { Society of } \\
\text { Resource } \\
\text { Geology }\end{array}$ & Japón \\
\hline Revista Geológica de Chile & 0716-0208 & 112 & 0.321 & 0.143 & 14 & 8.4 & $\begin{array}{c}\text { Servicio } \\
\text { Nacional de } \\
\text { Geología y } \\
\text { Minería }\end{array}$ & Chile \\
\hline $\begin{array}{l}\text { Revista Mexicana de } \\
\text { Ciencias Geológicas }\end{array}$ & $1026-8774$ & 53 & 0.230 & 0.080 & 25 & 2.4 & UNAM & México \\
\hline $\begin{array}{l}\text { Rivista Italiana di } \\
\text { Paleontologia e Stratigrafia }\end{array}$ & $0035-6883$ & 262 & 0.292 & 0.125 & 16 & $>10.0$ & $\begin{array}{l}\text { Università degli } \\
\text { Studi di Milano }\end{array}$ & Italia \\
\hline $\begin{array}{l}\text { SPE Reservoir Evaluation } \\
\text { and Engineering }\end{array}$ & $1094-6470$ & 65 & 0.197 & 0.017 & 59 & & $\begin{array}{l}\text { Society of } \\
\text { Petroleum } \\
\text { Engineers }\end{array}$ & EUA \\
\hline
\end{tabular}

Letters (Tabla 2), ambas consideradas de frontera y con factores de impacto parecidos, sin embargo la primera tiene un índice de inmediatez más bajo debido posiblemente a que su periodicidad es más baja. En la Tabla 1 es posible detectar que las revistas de Geología Regional tienen un índice de inmediatez muy bajo, lo que puede tener su explicación debido a la baja periodicidad y a que no se consideran revistas de frontera.

\subsubsection{Vida media citada}

La vida media citada es el número de años de publicación, contados a partir del presente año, en los que se suma el $50 \%$ de las citas recibidas hasta la fecha. Este indicador ayuda a evaluar la edad de la mayoría de los artículos citados en una revista. La vida media de varias revistas de geociencias es mostrada en la Tabla 1. El ISI únicamente calcula la vida media citada a las revistas que han sido citadas más de cien veces.

La RMCG tuvo en el 2002 una vida media de 2.4 años; se puede destacar que en ese año se capturaron 53 citas, las cuales representan el $24 \%$ del total de las citas recibidas. Las citas capturadas en el 2001 más las del 2002, representan el $45 \%$ y sumando las del 2000 son el $62 \%$ del total. Es decir en los últimos 2.4 años se ha recibido el $50 \%$ de las citas de la RMCG. La vida media del 2001 fue de 2 años.

Según el ISI, un alto o bajo valor de vida media citada no implica ningún valor particular de una revista. Por ejemplo, una revista de investigación básica (primaria) puede tener una vida media más larga que una revista que ofrece una rápida comunicación de información actual. El
ISI propone que la vida media puede ser útil para ayudar en las decisiones del manejo y archivo de la colección, ya que los cambios dramáticos en la vida media citada a lo largo del tiempo puede indicar un cambio en el formato de la revista.

En el caso de la RMCG, el valor bajo de la vida media citada se debe a los cambios en sus políticas editoriales y de distribución. La vida media citada muestra que en los últimos años ha aumentado considerablemente su número de citas del SCI con respecto a sus etapas anteriores.

\subsubsection{Lista de revistas que la citan}

El Journal Citation Report (JCR) del ISI identifica aquellas publicaciones que más frecuentemente citan una revista en particular. Esta relación puede revelar la orientación del tema de la revista, apuntando a su competidor más cercano y describe redes de especialidades entre revistas.

Las revistas que más citan a la RMCG obtenidas del JCR son mostradas en la Tabla 3. Las diez revistas que más citan a las revistas de Geología de Centro y Sudamérica son mostradas en la Figura 6. Es posible observar que la RMCG tiene un fuerte componente de Geología Regional de Norteamérica, Vulcanología y Paleontología. En contraste, el Journal of South American Earth Sciences y la Revista Geológica de Chile tienen un alto componente de la Geología de Sudamérica. Se observa que cerca del $25 \%$ de las citas corresponden a autocitas (citas de esa misma revista).

De la Figura 6 también se desprende que la RMCG es 


\section{Revistas cuya editorial no es una empresa privada}

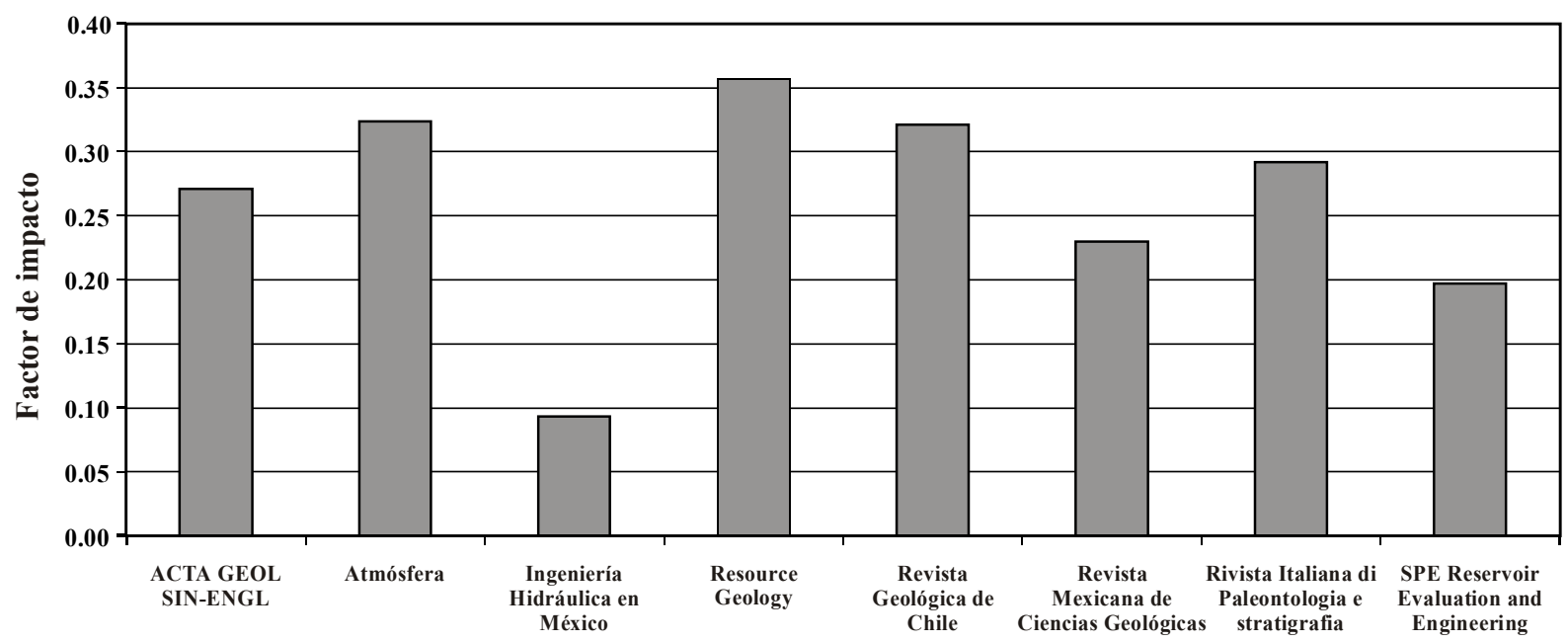

Figura 5. Comparación del factor de impacto (FI) 2002 de la RMCG con el FI 2001 de revistas de Geología Regional contenidas en el SClexpanded. Se utilizaron años distintos debido a que durante la búsqueda en el JCR, a principios del 2003, no estaban disponibles los datos del 2002 y no se utilizó el FI 2001 de la RMCG porque fue anómalamente bajo.

citada en revistas de alto impacto y gran prestigio en Ciencias de la Tierra. Creemos que esto se debe posiblemente a dos factores: (1) la cercanía y la colaboración que existe entre los científicos mexicanos y sus pares de Estados Unidos y Canadá, lo que da difusión a los trabajos publicados en la RMCG, y (2) que muchos científicos mexicanos publican en esas revistas y citan los trabajos de la RMCG, donde comúnmente se encuentran publicados datos fundamentales de la geología de México.

\section{Discusión}

\subsection{Importancia de la revista en la sociedad}

En las evaluaciones académicas se le ha dado un valor específico grande a las revistas del SCI, ya que se considera que cuentan con estándares de edición e impacto internacionales altos. En el supuesto de que la importancia de cada investigación depende del uso que se le dé al conocimiento generado por parte de otros individuos, se ha establecido que la manera más fácil de calificar un trabajo científico es a través de las citas recibidas. Extrapolando esta idea, el factor de impacto de una revista será un indicador de su calidad, para lo cual se requiere primeramente su inclusión en el SCI. Los artículos publicados en revistas no incluidas en este índice no son considerados en las evaluaciones o se les atribuye muy poco valor. A este respecto, hay que hacer notar que el $15 \%$ de las revistas incluidas en el SCI, en la categoría de Geología, tienen un factor de impacto igual o menor al obtenido por la RMCG.

Más allá del valor que puedan tener las citas, los trabajos geológicos publicados tienen valor por su utilidad en el desarrollo de una sociedad, ya que aportan información que es directamente utilizada por diversos sectores industriales y de servicios. Al igual que en otras ciencias naturales, el primer paso en la investigación geológica consiste en la obtención de los datos fundamentales, tanto cualitativos como cuantitativos, obtenidos a partir de la observación del medio natural y la medición de parámetros, realizada en el campo o en laboratorio. Típicamente esta parte del trabajo consiste en la cartografía geológica, el establecimiento de las relaciones estratigráficas de las unidades de roca, la obtención de la composición química de las rocas, la medición de estructuras, la determinación de edades relativas y absolutas de unidades de roca, minerales o estructuras, la descripción y clasificación de fósiles, la interpretación de los paleoambientes, etc. A partir de este conocimiento es posible hacer inferencias acerca de los mecanismos que dan lugar a los procesos geológicos. En cada proceso geológico interviene un número muy grande de variables, cuyas relaciones comúnmente no son lineales. La reconstrucción de los fenómenos geológicos se basa, en su primera aproximación, en la interpretación de las observaciones a la luz del "principio de las causas presentes", el cual establece que los fenómenos observados actualmente en la naturaleza, son iguales o muy semejantes a los ocurridos en el pasado geológico. Así pues, es claro que la descripción de las unidades de roca y de las estructuras es necesaria para establecer el cuándo y cómo ocurrieron los fenómenos en el tiempo y en el espacio. En lugares como México esa información fundamental es aún muy incompleta.

Por lo anterior, es posible afirmar que el acopio de información descriptiva y la interpretación de la evolución de los sistemas geológicos en escalas locales y regionales, son una parte insoslayable del quehacer de las geociencias 
Tabla 2. Ejemplo de dos revistas de geociencias con altos indicadores de impacto.

\begin{tabular}{lccccc}
\hline Título de la revista & ISSN & $\begin{array}{c}\text { Total de citas } \\
\mathbf{2 0 0 1}\end{array}$ & $\begin{array}{c}\text { Factor de } \\
\text { Impacto }\end{array}$ & $\begin{array}{c}\text { Índice de } \\
\text { inmediatez }\end{array}$ & $\begin{array}{c}\text { Artículos } \\
\mathbf{2 0 0 1}\end{array}$ \\
\hline $\begin{array}{l}\text { Earth and Planetary Science } \\
\text { Letters }\end{array}$ & $0012-821 \mathrm{X}$ & 14885 & 2.7 & 0.38 & 355 \\
Geology & $0091-7613$ & 11440 & 2.488 & 0.292 & 305 \\
\hline
\end{tabular}

en nuestro país y constituyen en sí mismos activos intelectuales que deben estar accesibles a la comunidad nacional.

\subsection{Importancia de la revista con respecto al lector}

Las citas se consiguen de manera más oportuna si el trabajo se publica en la revista adecuada ya que la leerán las personas interesadas en el tema. Pongamos como ejemplo los distintos tipos de auditorio que puede tener el estudio de las relaciones entre volcanismo y deformación en la corteza superior terrestre (fallamiento).

\subsubsection{Publicaciones tipo A: Revistas nacionales que cumplen estándares internacionales.}

La descripción detallada de las relaciones espaciales y temporales entre las fallas y las rocas volcánicas de una región en particular, puede ser de interés para todo aquel que quiera estudiar esa región desde cualquier punto de vista. Esa información deberá ser publicada en una revista a la que tengan acceso las personas que potencialmente puedan requerirla. Por ejemplo, la información geológica básica puede ser útil para un gobierno estatal con el fin de cuantificar los recursos naturales o en el desarrollo de programas de protección civil; para profesionistas geólogos, geofísicos, ingenieros civiles, será útil en la construcción de obras civiles; y como información básica para investigadores de otras disciplinas que trabajen en la misma región: sismólogos, geodestas, geógrafos. Este tipo de trabajo es publicado en revistas nacionales como la Revista Mexicana de Ciencias Geológicas, Geofísica Internacional, el Boletín de la Sociedad Geológica Mexicana, GEOS, Boletín de la Asociación Mexicana de Geólogos Petroleros, etc.; ejemplos de trabajos publicados sobre las relaciones entre el fallamiento y volcanismo en México son: MartínBarajas (2000), Alaniz-Álvarez (2001), Morán-Zenteno et al. (1998). No suena sensato publicar este tipo de información en revistas de geología regional de otros países y menos aún en otros idiomas, ya que quedaría fuera del alcance de los profesionistas nacionales, quienes difícilmente tienen acceso a esas publicaciones.

\subsubsection{Publicaciones tipo B. Revistas internacionales con estándares internacionales de edición.}

La interrelación entre distintos tipos de procesos geológicos es estudiada desde varios puntos de vista por la comunidad geológica internacional. Es por esto que la descripción detallada de la geología de una región puede ser publicada en revistas de Geología de cualquier parte del mundo (e.g., Geological Society of America Bulletin, Candian Journal of Earth Sciences, Journal of South American Earth Sciences, entre otras) siempre y cuando se presente un enfoque novedoso o que tenga implicaciones interregionales de gran escala. En revistas con difusión global enfocadas a temas particulares de la Geología (Tectonics, Journal of Geophysical Research, Tectonophysics, Journal of Volcanology and Geothermal Research) se presentan también los modelos analógicos o geológicos y su aplicación en ciertas regiones. Si se establece el modelo teórico de cómo opera un fenómeno geológico, entonces el trabajo debe publicarse en una revista especializada (Bulletin of Vulcanology, Journal of Structural Geology). Así, con respecto a las relaciones entre el volcanismo y el fallamiento, se pueden citar algunos ejemplos: se publicó en el Geological Society of America Bulletin un trabajo sobre la actividad volcánica en Camargo, Chih. (Aranda-Gómez et al., 2003), en Tectonophysics un modelo analógico de la relación entre el tipo de volcán y el fallamiento (Takada, 1994), en Tectonics, la explicación de porqué una zona con alta actividad volcánica no tiene la misma cantidad de fallas que las zonas aledañas con menos volcanismo (Parsons et al., 1998).

\subsubsection{Publicaciones tipo $C$ : Revistas de frontera.}

$\mathrm{Si}$ el tema es de vanguardia, son ideas novedosas, hay mucha expectativa en el curso de ese tipo de investigación, es la primera explicación de un fenómeno o el primer hallazgo a nivel mundial, se podrá publicar en revistas que ofrecen una difusión global rápida como Nature o Science y en geociencias como Geology y Earth and Planetary Science Letters. Para el caso que ejemplificamos, se ha publicado la explicación teórica de cómo el volcanismo puede inhibir la sismicidad en una región (Parsons and Thompson, 1991), el cuestionamiento de las plumas como el origen del magmatismo de intraplaca en las islas Australes (McNutt et al., 1997), o el hallazgo de volcanismo en Marte (McEwen, et al., 1999).

En general, que el artículo corresponda o no a una revista en particular es una decisión de los editores, los cuales definen cuál es el público de cada revista. Sin embargo, en vista de que para los investigadores mexicanos es más conveniente publicar en una revista del SCI ya que son mejor calificados en los esquemas de evaluación dominantes en 
México, la publicación de los datos de geología básica (fundamental) de México, pudiendo corresponder a publicaciones tipo A, se tratan de orientar hacia revistas extranjeras (tipo B) de interés para un público internacional (e.g., Aranda-Gómez et al., 2003; Morán-Zenteno et al., 1999, Talavera-Mendoza y Suastegui, 2000; Nagy et al., 1999). Esto es visto con buenos ojos en el sistema científico mexicano debido al reconocimiento internacional de la publicación, lo cual no tendría nada de malo, si no es porque se deja afuera a gran parte del público nacional potencialmente interesado y que no tiene acceso a la mayoría de estas revistas, ya sea por razones de idioma, de escasez en bibliotecas, o por su alto costo. Considérese que el precio institucional de la suscripción anual de las revistas de Geología varía de 4,500 a 49,000 pesos (Tabla 3). Además, al adquirir esas publicaciones el profesionista (privado o gubernamental) se daría cuenta de que los artículos con información de su interés, relacionada con el entorno geológico nacional o de la región donde trabajan, son esporádicos. Por otra parte, al publicar en revistas internacionales tipo B la información geológica de nuestro territorio tiende a dispersarse entre las numerosas revistas de geología de Norteamérica, o bien en las revistas especializadas. Considérese como prueba de esto que si se divide el número de artículos sobre la Geología de México en las revistas internacionales que aparecen en el SCI, entre el número total de artículos de dicha revista la razón obtenida es mínima. Esto muestra que el impacto de la comunidad geológica nacional es insignificante a escala global; sin embargo es potencialmente muy importante para el país.

Tabla 3. Lista de revistas incluidas en el SCIe que más citan a la RMCG

\begin{tabular}{|c|c|c|c|}
\hline TÍTULO & $\begin{array}{c}\text { Número de citas } \\
\text { de 1996-2002 }\end{array}$ & $\begin{array}{c}\text { Factor de } \\
\text { impacto (2001) }\end{array}$ & $\begin{array}{l}\text { Precio en Dólares } \\
\text { para instituciones }\end{array}$ \\
\hline J. S. AM. EARTH SCI. & 17 & 0.681 & 858 \\
\hline J. VOLCANOL. GEOTHERM. RES. & 16 & 1.003 & 2580 \\
\hline INT. GEOL. REV. & 14 & 0.767 & 997 \\
\hline GEOL. SOC. AM. BULL. & 14 & 2.249 & 485 \\
\hline GEOLOGY & 13 & 2.488 & 485 \\
\hline J. PALEONTOL. & 13 & 0.812 & 116 \\
\hline TECTONOPHYSICS & 10 & 1.473 & 4399 \\
\hline SPECIAL PALAEONTOL. & 7 & & \\
\hline PALAEOGEO. PALAEOCLIMATOL. PALAEOECOL. & 5 & 1.449 & 3353 \\
\hline CRET. RES. & 5 & 0.795 & 825 \\
\hline BULL. SOC. GEOL. FR. & 5 & 0.630 & $125^{*}$ \\
\hline J. FORAM. RES. & 4 & 1.236 & 90 \\
\hline TECTONICS & 3 & 2.526 & 528 \\
\hline NEUES JARHB. GEOL. PALAEONTOL. A & 3 & 0.466 & 277.2 \\
\hline C. R. ACAD. SCI. II. A & 3 & 0.366 & 725 \\
\hline J. GEOPHYS. RES. B. SOLID EARTH & 2 & 2.609 & 3000 \\
\hline BULL. VOLCANOL. & 2 & 1.671 & $789.80 *$ \\
\hline SEDIM. GEOL. & 2 & 1.166 & 2688 \\
\hline EPISODES & 2 & 0.944 & 24 \\
\hline PALAEONTOLOGY & 2 & 0.731 & 612 \\
\hline GEOBIOS & 2 & 0.615 & 155 \\
\hline AAPG BULL. & 2 & 1.173 & 305 \\
\hline CONTRIB. MINERAL. PETROL. & 1 & 2.534 & $2898.2 *$ \\
\hline ECON. GEOL. & 1 & 1.368 & 145 \\
\hline INT. J. EARTH SCI. & 1 & 1.375 & $769.7 *$ \\
\hline MICROPALEONTOLOGY & 1 & 0.472 & 360 \\
\hline GEODERMA & 1 & 1.467 & 2172 \\
\hline FACIES & 1 & 0.911 & $150^{*}$ \\
\hline GONDWANA RES. & 1 & 0.548 & 130 \\
\hline J. PETROL. GEOL. & 1 & 0.537 & 360 \\
\hline ING. HIDRAUL. MEX. & 1 & 0.093 & 50 \\
\hline Total & 155 & & \\
\hline
\end{tabular}

\footnotetext{
* en Euros
} 
a)

\section{Revista Mexicana de Ciencias Geológicas}

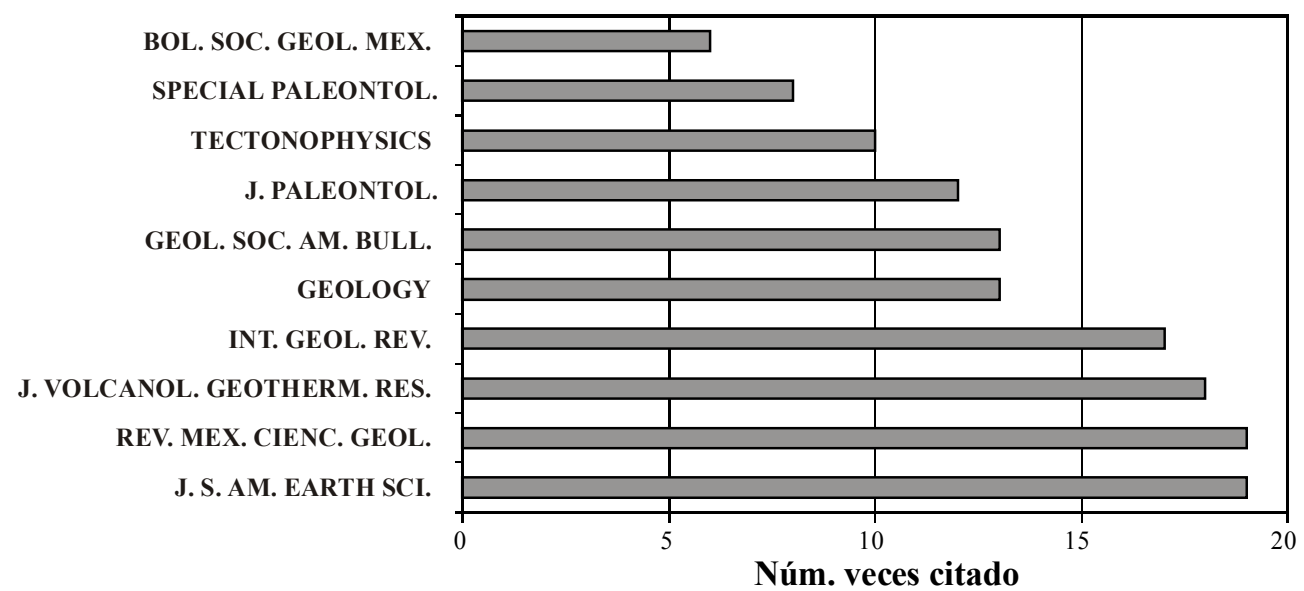

b)

\section{Journal of South American Earth Sciences}

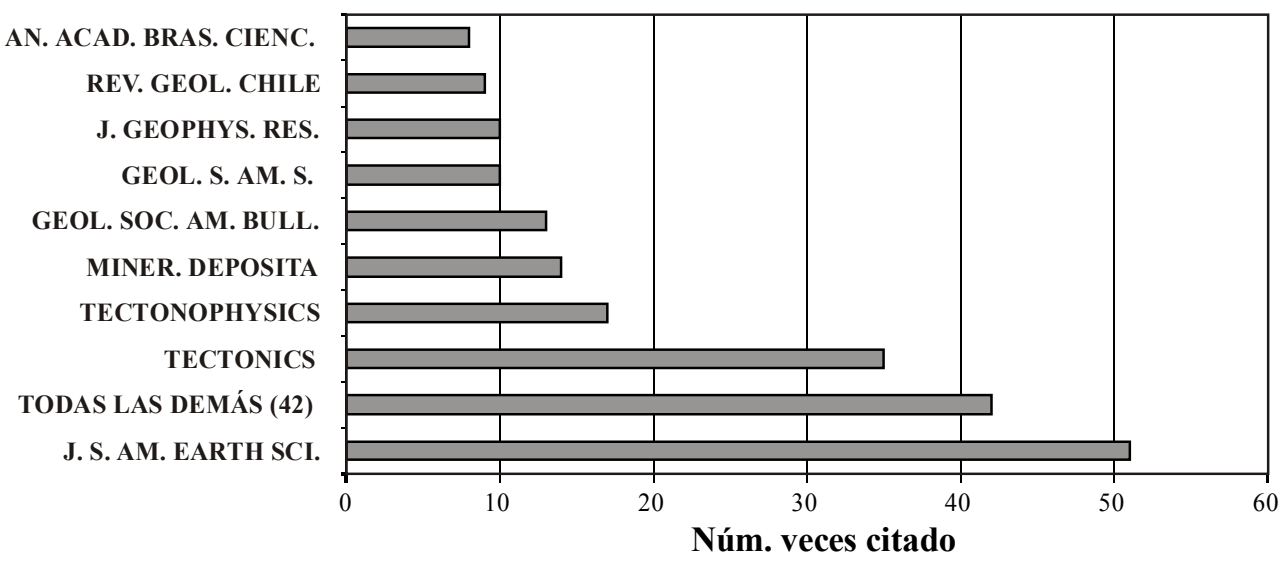

c)

Revista Geológica de Chile

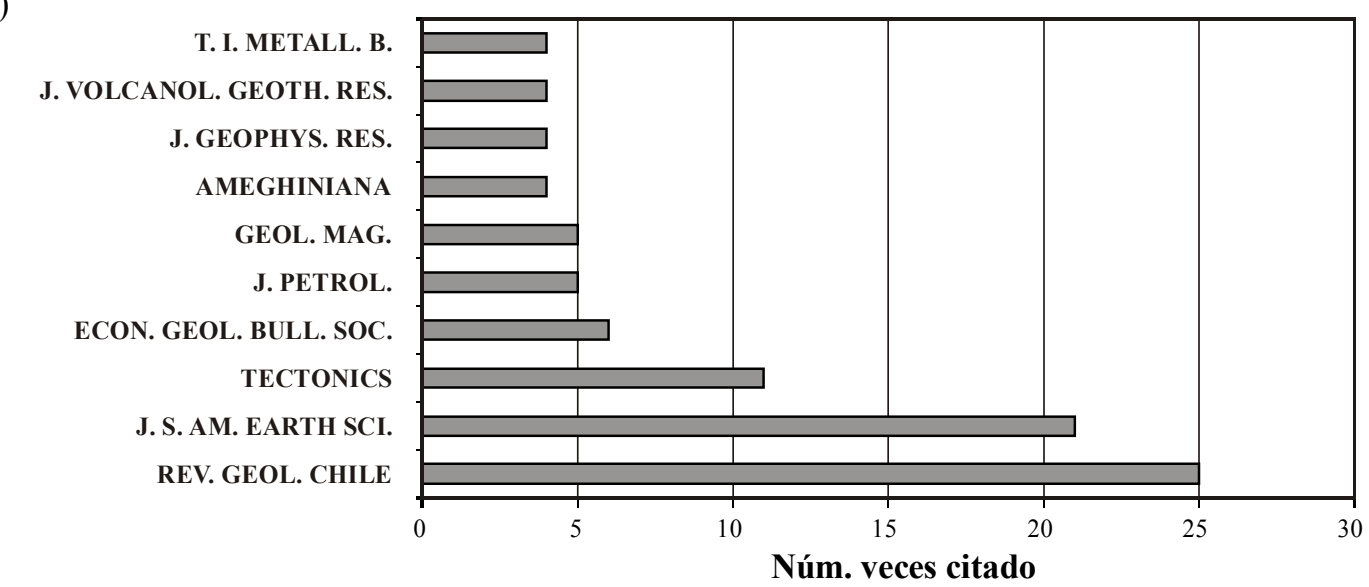

Figura 6. Lista de las diez revistas que más citan a (a) RMCG (Revista Mexicana de Ciencias Geológicas, b) Journal of South American Earth Sciences y c) Revista Geológica de Chile. 


\subsection{Vinculación entre ciencia, gobierno e industria}

En México se ha insistido en la necesidad de establecer vínculos entre la industria, el gobierno y la ciencia. Con este propósito se han mencionado las ventajas de efectuar contratos o convenios entre ambos, con lo cual una parte de los gastos del quehacer científico nacional serían absorbidos por instituciones privadas, disminuiría la aportación del gobierno federal al subsidio de las universidades y el trabajo científico sería en beneficio de la sociedad. Por otra parte, se ha insistido en que los investigadores publiquemos en las revistas incluidas en el SCI, es decir, revistas certificadas por el ISI como revistas que tienen influencia en el desarrollo de la investigación actual.

Los geólogos que nos dedicamos a la investigación científica nos enfrentamos al reto de cumplir las dos expectativas: convencer a la industria y al gobierno que paguen por una investigación que no están acostumbrados a pagar y que ésta tenga impacto científico internacional.

Proponemos que en Geología, y seguramente en muchas otras disciplinas, se puede establecer una vía de comunicación entre el gobierno, la industria y la academia para el intercambio de información. Esta vía son las publicaciones nacionales con estándares internacionales de edición, ya que estas revistas son completamente accesibles a la comunidad nacional y contienen un componente científico de alto nivel.

\section{Conclusiones}

En el sistema científico mundial se ha hecho la división entre las revistas incluidas en el SCI y las no incluidas, asumiendo que las primeras siempre son mejores que las segundas. En este trabajo se muestra que la RMCG cumple con los estándares internacionales de edición y se ofrecen los valores obtenidos de los indicadores del ISI para sustentarlo; de esta manera la revista puede ser calificada objetivamente.

La existencia de una revista científica mexicana sobre Geología es importante para el país, no sólo por el papel que tiene en la vinculación de los sectores académico, gubernamental y privado, sino también porque promueve y fomenta la actividad intelectual de alto nivel científico en el interior de nuestro país.

\section{Agradecimientos}

Se agradecen los múltiples comentarios hechos por Saúl Armendáriz y Margarita Lugo acerca de la importancia de las revistas científicas nacionales, los cuales motivaron en gran parte escribir este artículo. Los comentarios de Dante J. Morán y Claudia Mendoza ayudaron a mejorar el contenido de este artículo.

\section{Referencias bibliográficas}

Alaniz-Álvarez, S.A., Nieto-Samaniego, A.F., Reyes-Zaragoza, M.A., Orozco-Esquivel, M.T., Ojeda-García, A.C., Vassallo, L., 2001, Estratigrafía y deformación extensional en la región San Miguel de Allende-Querétaro, México: Revista Mexicana de Ciencias Geológicas, 18, 129-148.

Aranda-Gomez, J.J, Luhr, J.F, Housh, T.B, 2003, Synextensional PliocenePleistocene eruptive activity in the Camargo volcanic field, Chihuahua, Mexico: Geological Society of America Bulletin, 115, 298-313.

Martín-Barajas, A., 2000, Volcanismo y extensión en la provincia extensional del Golfo de California: Boletín de la Sociedad Geológica Mexicana, 53, 72-83.

McEwen, A.S., Malin, M.C., Carr, M.H., Hartmann, W.K., 1999, Voluminous volcanism on early Mars revealed in Valles Marineris: Nature, 397, 584-586.

McNutt, M.K., Caress, D.W., Reynolds, J., Jordahl, K.A.. Duncan, R.A., 1997, Failure of plume theory to explain midplate volcanism in the southern Austral islands: Nature, 389, 479-482.

Morán-Zenteno, D.J., Tolson, G., Alba-Aldave, L., Martínez-Serrano, R.G., Reyes-Salas, M.A., Corona-Esquivel, R., Ángeles-García, S., 1998, Stratigraphy, geochemistry and tectonic significance of the Tertiary volcanic sequences of the Taxco-Quetzalapa region, southern Mexico: Revista Mexicana de Ciencias Geológicas, 15, 167-180.

Morán-Zenteno, D.J., Tolson, G., Martínez-Serrano, R.G., Martiny, B., Schaaf, P., Silva-Romo, G., Macías-Romo, C., Alba-Aldave, L., Hernández-Bernal, M.S., Solís-Pichardo, G.N., 1999, Tertiary arcmagmatism of the Sierra Madre del Sur, Mexico, and its transition to the volcanic activity of the Trans-Mexican Volcanic Belt: Journal of South American Earth Sciences, 12, 513-535.

Nagy, E.A., Grove M., Stock, J.M. 1999, Age and stratigraphic relationships of pre- and syn-rift volcanic deposits in the northern Puertecitos Volcanic Province, Baja California, Mexico: Journal of Volcanology and Geothermal Research, 93, 1-30.

Parsons, T., Thompson, G.A., 1991, The role of magma overpressure in suppressing earthquakes and topography; worldwide examples: Science, 253, 1399-1402.

Parsons, T., Thompson, G.A., Smith, R.P., 1998, More than one way to stretch: a tectonic model for extension along the plume track of the Yellowstone hotspot and adjacent Basin and Range Province: Tectonics, 17, 221-234.

Sylvan-Katz, J., 1999, The self-similar science system: Research Policy, 28, 501-517.

Takada, A., 1994, The influence of regional stress and magmatic input on styles of monogenetic and polygenetic volcanism: Journal of Geophysical Research, 99, 13563-13573.

Talavera-Mendoza, O., Suastegui, M.G., 2000, Geochemistry and isotopic composition of the Guerrero Terrane (western Mexico): implications for the tectono-magmatic evolution of southwestern North America during the late Mesozoic: Journal of South American Earth Sciences, $13,297-324$.

Testa, J., 2003, The ISI ${ }^{\circledR}$ database: the journal selection process: http:// sunweb.isinet.com/isi/hot/essays/selectionofmaterialforcoverage/ 199701.html, consultado el 4 de Septiembre, 2003, 20:00 hrs.

Manuscrito recibido: Junio 26, 2003

Manuscrito corregido recibido: Noviembre 3, 2003

Manuscrito aceptado: Noviembre 10, 2003 